Article

\title{
Study of Current Measurement Method Based on Circular Magnetic Field Sensing Array
}

\author{
Zhenhua Li ${ }^{1,2, *}$, Siqiu Zhang ${ }^{1}$, Zhengtian $\mathrm{Wu}^{3}{ }^{3}$, Ahmed Abu-Siada 4 (D) and Yuan Tao ${ }^{1}$ \\ 1 College of Electrical Engineering \& New Energy, China Three Gorges University, Yichang 443002, China; \\ zhangsiqiu1208@163.com (S.Z.); taoyuan2016ty@163.com (Y.T.) \\ 2 Hubei Provincial Collaborative Innovation Center for New Energy Microgrid, \\ China Three Gorges University, Yichang 443002, China \\ 3 School of Electronic and Information Engineering, Suzhou University of Science and Technology, \\ Suzhou 215009, China; wzht8@mail.usts.edu.cn \\ 4 Electrical and Computer Engineering Department, Curtin University, Perth 6000, WA, Australia; \\ A.AbuSiada@exchange.curtin.edu.au \\ * Correspondence: lizhenhua1993@163.com; Tel.: +86-188-7172-6917
}

Received: 9 April 2018; Accepted: 3 May 2018; Published: 5 May 2018

\begin{abstract}
Classic core-based instrument transformers are more prone to magnetic saturation. This affects the measurement accuracy of such transformers and limits their applications in measuring large direct current (DC). Moreover, protection and control systems may exhibit malfunctions due to such measurement errors. This paper presents a more accurate method for current measurement based on a circular magnetic field sensing array. The proposed measurement approach utilizes multiple hall sensors that are evenly distributed on a circle. The average value of all hall sensors is regarded as the final measurement. The calculation model is established in the case of magnetic field interference of the parallel wire, and the simulation results show that the error decreases significantly when the number of hall sensors $n$ is greater than 8 . The measurement error is less than $0.06 \%$ when the wire spacing is greater than 2.5 times the radius of the sensor array. A simulation study on the off-center primary conductor is conducted, and a kind of hall sensor compensation method is adopted to improve the accuracy. The simulation and test results indicate that the measurement error of the system is less than $0.1 \%$.
\end{abstract}

Keywords: circular array; magnetic field sensor; current measurement; magnetic interference

\section{Introduction}

Instrument transformer is one of the key assets in the substations as it affirms the reliability of the protection and control systems [1-4]. The conventional magnetic core-based transformer is subject to saturation that limits its ability to measure direct current (DC) $[5,6]$. On the other hand, an electronic instrument transformer based on Rogowski coil is widely used in power systems due to its wide dynamic range and low cost. However, it cannot be used for DC measurement because of its sensing principle [7-10]. While an optical transformer can measure both alternating current (AC) and DC signals, the temperature stability and mechanical properties of optical crystals result in the low measurement accuracy of this instrument [11-16]. Commonly used methods for measuring DC current include shunt methods, magneto-resistance, and magneto-optical effects. However, the shunt methods are bulky, which renders them unsuitable for onsite applications. Furthermore, the accuracy of measurement methods based on giant magneto-resistance and magneto-optical effects is not high as it depends on the stability of optical and magnetic materials [17-20]. Owing to the rapid development of semiconductor materials and power electronic technology, magnetic field sensors, especially hall 
sensors, have attracted much attention for DC current measurement [21-27]. As there is no iron core, the hall sensor is not susceptible to magnetic saturation and can be used to measure large DC currents.

To overcome the deficiency of conventional transformers and improve the accuracy of DC measurement methods, this paper proposes a current measurement method based on a circular magnetic field sensing array. Circular magnetic field sensing arrays were previously investigated in References [28,29]. However, these investigations mainly focused on signal processing algorithms. When there is the interference of an external current, an algorithm based on spatial Discrete Fourier Transform (DFT) was proposed to improve the interference rejection in Reference [28], and an algorithm based on the digital processing of a small set of magnetic field measurements was proposed to reduce the interference in Reference [29]. The method proposed in this paper is mainly focused on the sensor array structure and external interference factor, which improve the interference rejection by changing the number of sensors and the radius of sensor array. Moreover, a compensation method is proposed to improve the measurement accuracy when the conductor is off-center. The method employs eight hall sensors that are evenly distributed on a circle, in which the magnetic field strength can be measured and hence the current can be calculated. The interference from a parallel wire carrying the same current and an off-center wire are the dominant sources of error in practice. So, by using the average value of these hall sensors as the final measurement output, we can effectively reduce the external magnetic field interference and improve the accuracy. The simulation model is established and the simulation analysis is carried out when there is magnetic field interference from the parallel wire and off-center wire. The results show that the measurement error is less than $0.06 \%$ when the wire spacing is greater than 2.5 times the radius of the sensor array. By using a kind of compensation method for the hall sensor, the error generated by the off-center wire can be reduced effectively, and the whole system error is less than $0.1 \%$.

\section{Measurement Accuracy Analysis of Circular Magnetic Field Sensing Array}

The measurement method proposes several hall sensors distributed evenly on a circle, as shown in Figure 1 . The average value of all employed hall sensors is considered as the final measurement output. This arrangement can effectively reduce the external magnetic field interference and improve the measurement accuracy. The detailed analysis of the magnetic field interference of a parallel conductor is presented below. 


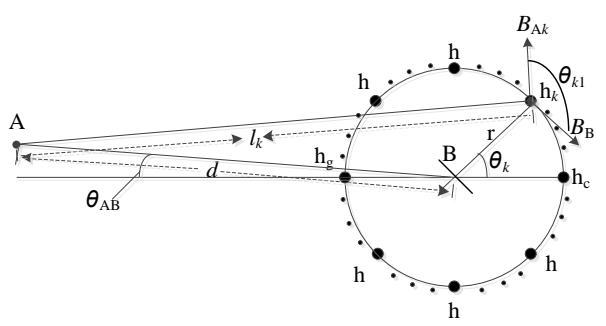

(a)

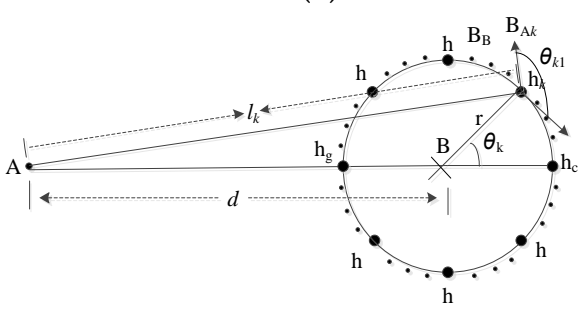

(b)

Figure 1. The magnetic field influence of a parallel conductor on the measurement accuracy of a circular hall sensors array: (a) conductor $\mathrm{A}$, conductor $\mathrm{B}, \mathrm{h}_{\mathrm{g}}$, and $\mathrm{h}_{\mathrm{c}}$ all are not on the same line; (b) conductor $\mathrm{A}$, conductor $\mathrm{B}, \mathrm{h}_{\mathrm{g}}$, and $\mathrm{h}_{\mathrm{c}}$ are on the same line.

As shown in Figure 1, the distance between the conductors A and B is assumed to be $d$, while the currents in both conductors are assumed to be in opposite direction and of values $I_{\mathrm{A}}$ and $I_{\mathrm{B}}$, respectively. Conductor $B$, in which the current is to be measured, coincides with the center of the circular hall sensor array. $n$-hall sensors are evenly distributed on a circle of radius $r$.

The hall sensors can only measure the magnetic field perpendicular to its sensitive area. The magnetic field density $B_{\mathrm{A} k}$ generated due to a current $I_{\mathrm{A}}$ passing in conductor $\mathrm{A}$ can be measured by the $k$ th hall sensor and is given by:

$$
B_{\mathrm{A} k}=\frac{\mu_{0} I_{\mathrm{A}}}{2 \pi l_{k}} \cdot \cos \theta_{k 1}
$$

where:

$$
\left\{\begin{array}{l}
l_{k}=\sqrt{\left(d \cos \theta_{A B}+r \cos \theta_{k}\right)^{2}+\left(r \sin \theta_{k}-d \sin \theta_{A B}\right)^{2}} \\
\cos \theta_{k 1}=-\frac{l_{k}^{2}+r^{2}-d^{2}}{2 l_{k} r}
\end{array}\right.
$$

From which $\cos \theta_{k 1}$ can be rewritten as:

$$
\cos \theta_{k 1}=-\frac{r+d \cos \left(\theta_{k}+\theta\right)}{l_{k}}
$$

$\left(\theta_{k}+\theta\right) \in(\theta, \theta+2 \pi)$, which means $\cos \left(\theta_{k}+\theta\right)$ and $r+d \cos \left(\theta_{k}+\theta\right)$ can be positive or negative; hence, $\cos \theta_{k 1}$ can be positive or negative. Adopting the average method would reduce the influence of the current in conductor $\mathrm{A}$ on the overall readings of the $n$ hall sensors.

The average magnetic field density value of $n$ hall sensors due to a current in conductor A can be obtained from (1) and (3), as given below:

$$
B_{\text {Aav }}=-\frac{\mu_{0} I_{\mathrm{A}}}{2 \pi n} \sum_{k=1}^{n} \frac{d \cos \left(\theta_{k}+\theta\right)+r}{d^{2}+r^{2}+2 d r \cos \left(\theta_{k}+\theta\right)}
$$


The average magnetic field density due to a current $I_{\mathrm{B}}$ in conductor $\mathrm{B}$ can be given as:

$$
B_{B a v}=\frac{\mu_{0} I_{\mathrm{B}}}{2 \pi r}
$$

The measurement error is:

$$
I_{e}=\frac{B_{A a v}}{B_{\text {Bav }}}=-\frac{r I_{\mathrm{A}}}{n I_{\mathrm{B}}} \sum_{k=1}^{n} \frac{d \cos \left(\theta_{k}+\theta\right)+r}{d^{2}+r^{2}+2 d r \cos \left(\theta_{k}+\theta\right)}
$$

The method proposed in Reference [28] is based on a spatial harmonic analysis of the magnetic field; the main content is about the analysis of the interference rejection algorithm. This method is useful to reduce the crosstalk relative error. It first calculates the magnetic scalar potential in a polar coordinate system, then the measurement error can be obtained by spatial Discrete Fourier Transform (DFT). In this paper, the main content concerns the error analysis of adjacent current, off-center influence, and the compensation method. From Equation (6), we determine that the measurement error is the ratio of the magnetic field generated due to the external current and the magnetic field generated due to the measured current. Moreover, the average method is adopted to reduce the interference of the external current.

When $I_{\mathrm{A}}$ and $I_{\mathrm{B}}$ are equal in magnitude:

$$
I_{e}=\frac{B_{\text {Aav }}}{B_{\text {Bav }}}=-\frac{r}{n} \sum_{k=1}^{n} \frac{d \cos \left(\theta_{k}+\theta\right)+r}{d^{2}+r^{2}+2 d r \cos \left(\theta_{k}+\theta\right)}
$$

In order to simplify the analysis, let the value of $\theta$ be $0^{\circ}$. Then:

$$
I_{e}=\frac{B_{A a v}}{B_{B a v}}=-\frac{r}{n} \sum_{k=1}^{n} \frac{d \cos \theta_{k}+r}{d^{2}+r^{2}+2 d r \cos \theta_{k}}
$$

where $\theta_{k}=\frac{2 k \pi}{n}, k=1,2 \ldots, n$.

The following simulation analysis is based on Equation (8).

\subsection{Correlation of the Measurement Error and the Number of Hall Sensors}

Considering the physical configuration of two practical conductors, $r$ is assumed to be in the range of $0.1 \mathrm{~m}-0.4 \mathrm{~m}$ and $d$ in the range of $1 \mathrm{~m}-3 \mathrm{~m}$. Simulation results for $r=0.1 \mathrm{~m}$ and $d=1.5 \mathrm{~m}$ are shown in Figure 2. It can be observed from Figure 2a that measurement error decreases substantially when the number of hall sensors increases. The error is less than $10^{-8}$ when $n \geq 8$. 


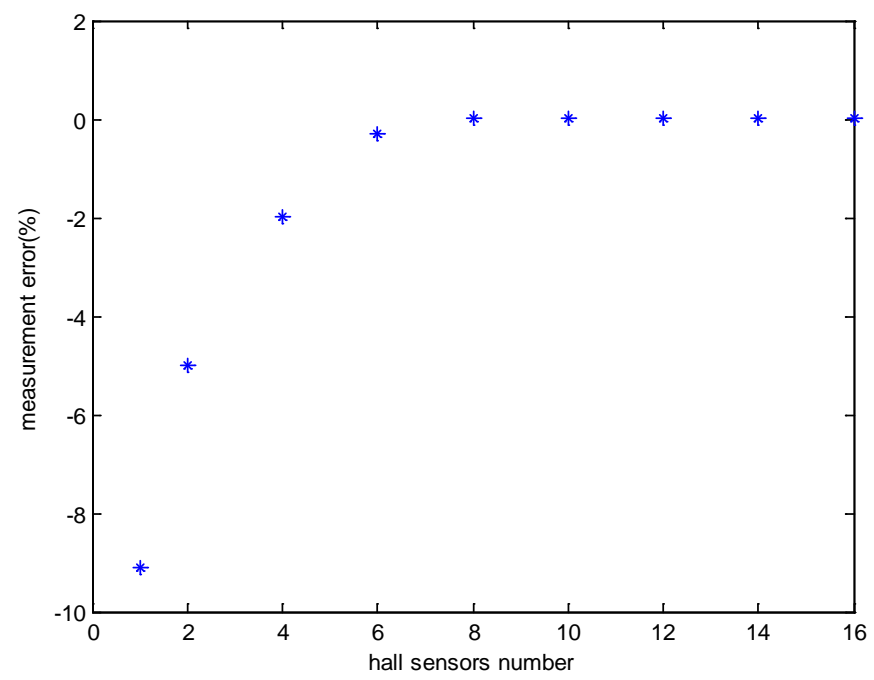

(a)

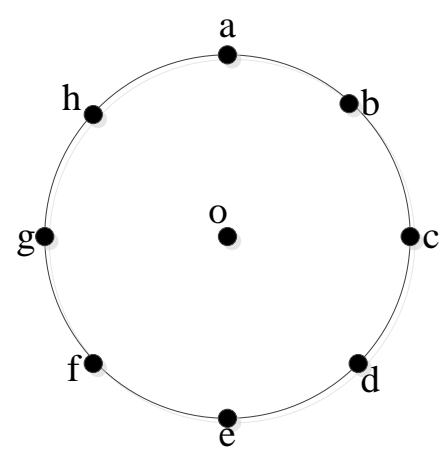

(b)

Figure 2. The measurement error and hall sensors position schematic: (a) the measurement error verses the hall sensors number; (b) the hall sensors position schematic. Hall sensors are distributed evenly in c and $\mathrm{g}$ with $n=2$, in $\mathrm{a}, \mathrm{c}, \mathrm{g}$, e with $n=4$, and in $\mathrm{a}, \mathrm{b}, \mathrm{c}, \mathrm{d}, \mathrm{e}, \mathrm{f}, \mathrm{g}$ with $n=8$.

\subsection{Correlation of the Measurement Error and the Distance between the Conductors}

In this study, $r$ is set to $0.1 \mathrm{~m}$ and $n$ is chosen to be 8 based on the investigation of the previous section. Figure 3 shows the measurement error for a wide range of the distance between the two conductors $(d)$. As can be seen in the figure, the error is less than $10^{-8}$ when the distance between the conductors is larger than $1 \mathrm{~m}$. 


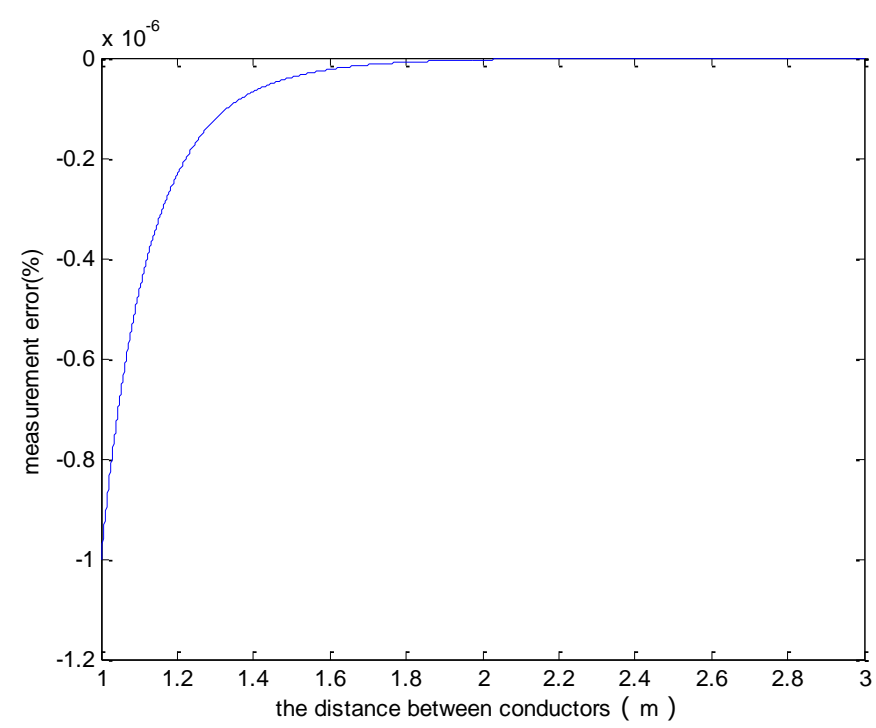

Figure 3. The measurement error verses the distance between the two conductors.

\subsection{Correlation of the Measurement Error and the Circle Radius}

This analysis is conducted for $d=1.5 \mathrm{~m}$ and $n=8$. Results shown in Figure 4 reveal that the measurement error is less than $0.003 \%$ for a circle radius less than $0.4 \mathrm{~m}$ and can be neglected.

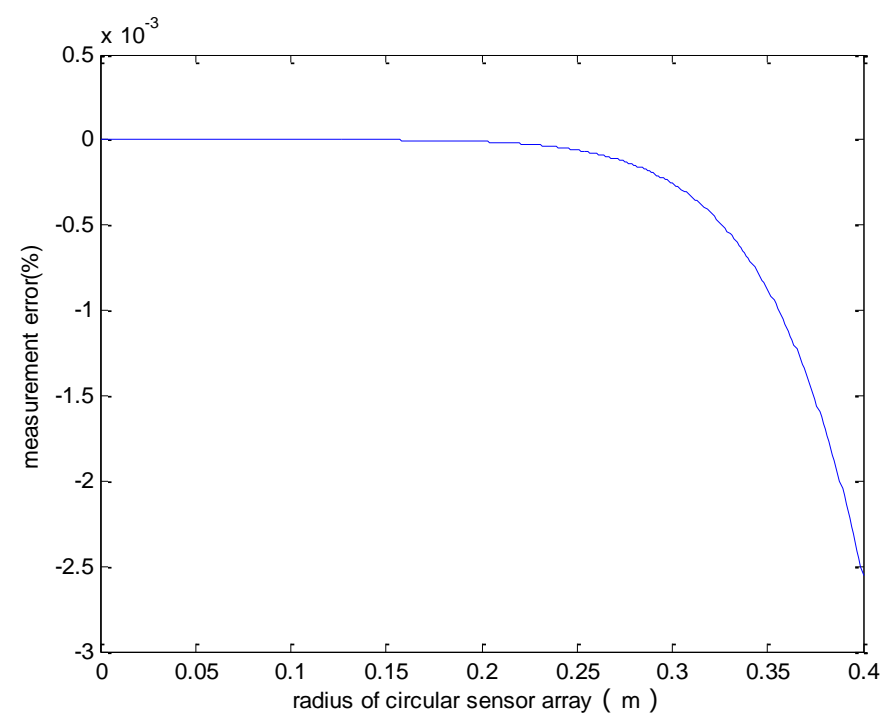

Figure 4. The measurement error verses the radius of the sensor array.

\subsection{Analysis of the Measurement Error with $d$ and $r$}

In order to facilitate the investigation of the influence of $r$ and $d$ on the measurement accuracy, Equation (8) is rewritten as:

$$
I_{e}=\frac{B_{A a v}}{B_{\text {Bav }}}=-\frac{1}{n} \sum_{k=1}^{n} \frac{\frac{d}{r} \cos \theta_{k}+1}{\left(\frac{d}{r}\right)^{2}+2 \frac{d}{r} \cos \theta_{k}}
$$

For $n=8$, the measurement error as a function of the $d / r$ ratio is shown in Figure 5 . It can be seen that the error is less than $0.05 \%$ when $d / r>2.5$. 


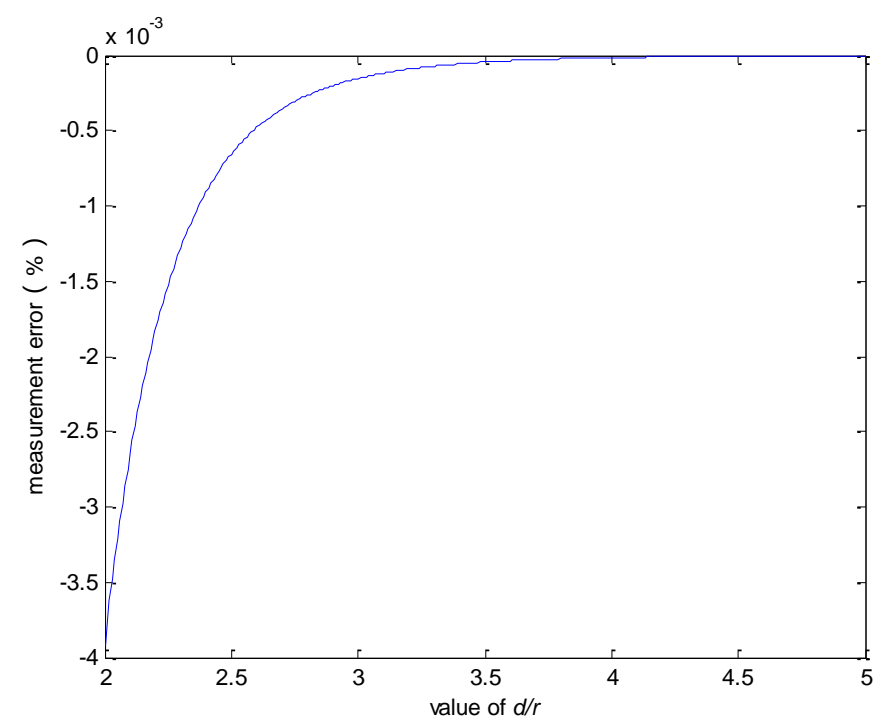

Figure 5. The measurement error verses the $d / r$ ratio.

From the above analysis, it can be seen that the measurement error is less than $0.05 \%$ when the number of sensors $n$ is larger than 8 , the distance between the conductors is 2.5 times the radius of sensor array, and $I_{\mathrm{A}}$ and $I_{\mathrm{B}}$ are equal in magnitude.

The average value of all hall sensors is used as the final measurement level. In this way, the effect of the external magnetic field interference can be reduced. Figures 6-8 present the output results of a single hall sensor for the three cases investigated above. Comparing these results with the measurement error based on the average of all hall sensors (Figures 3-5) reveals that by using the average value of the sensor array, the error can be effectively reduced.

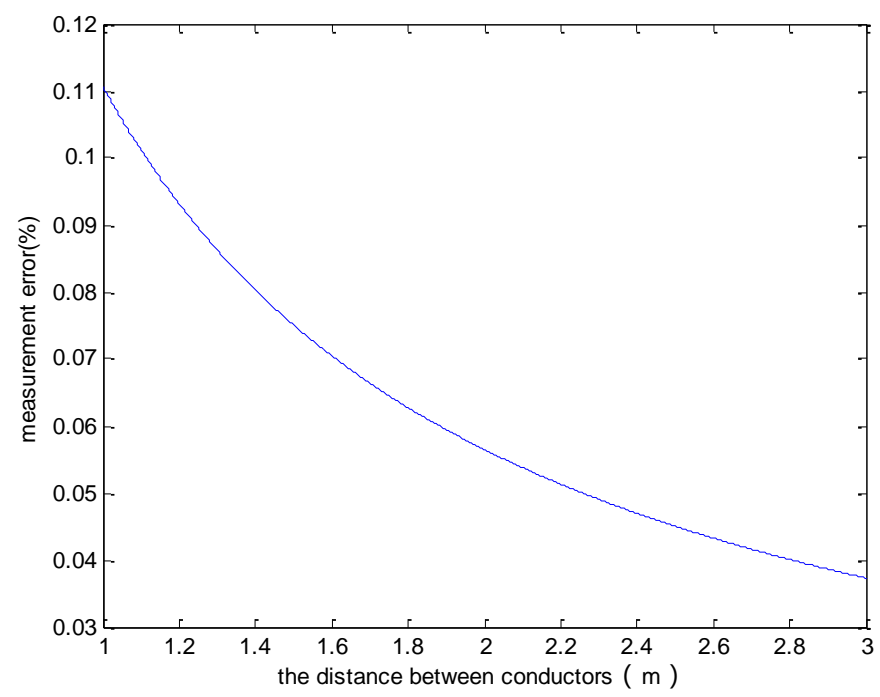

Figure 6. The measurement error of a single hall sensor verses the distance between conductors. 


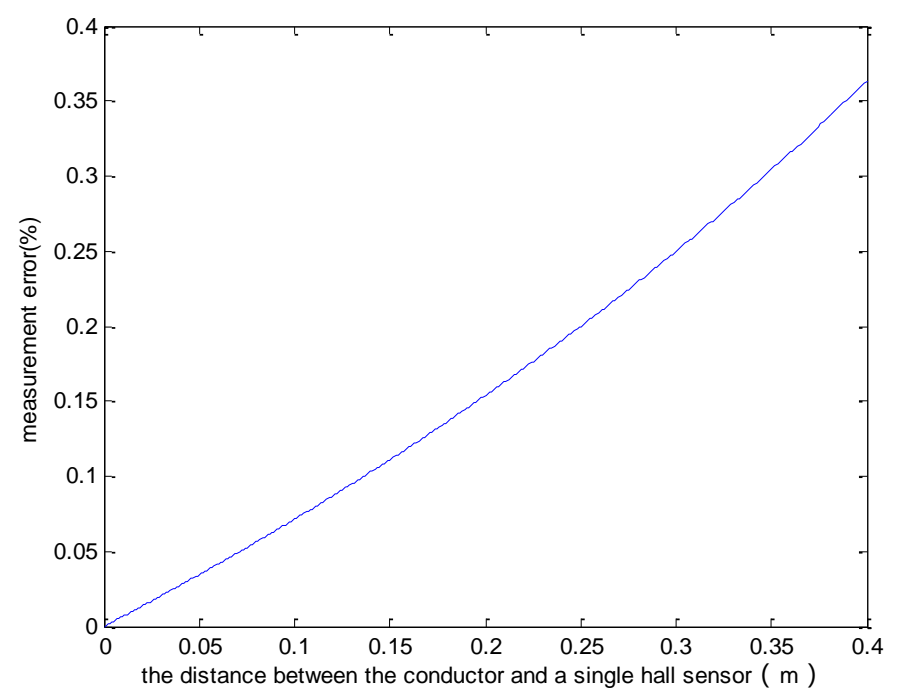

Figure 7. The measurement error of a single hall sensor verses the distance between the conductor and the sensor.

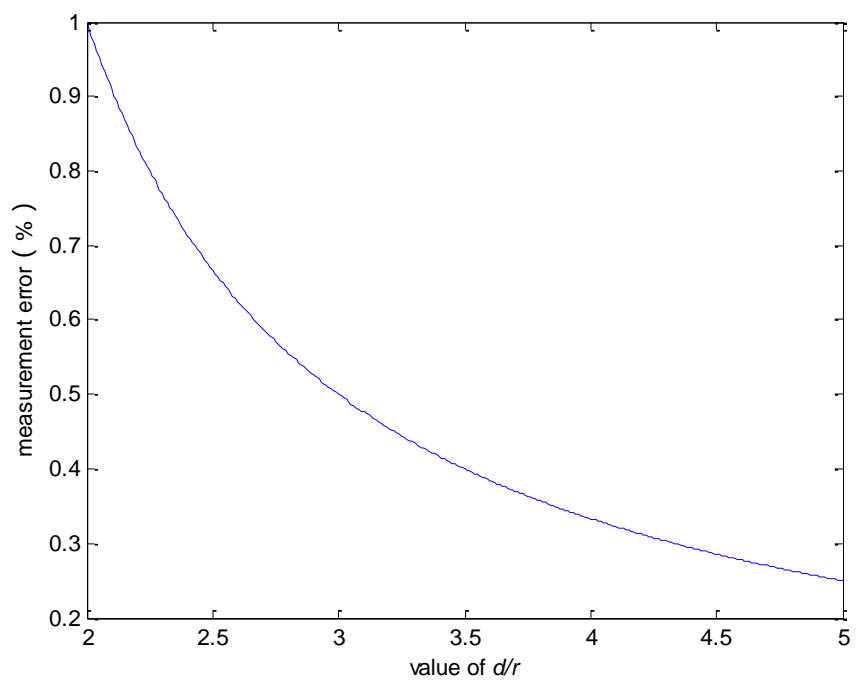

Figure 8. The measurement error of a single hall sensor verses the $d / r$ ratio.

\section{Error Analysis of Off-Center Distance}

The off-center position of the primary conductor is the most common problem that may lead to a significant measurement error when a current transformer is utilized to measure the current. A similar issue takes place when hall sensors are employed in this section; therefore, a compensation method of the magnetic field strength is proposed to improve the measurement accuracy of the hall sensors. The detailed analysis is presented below.

As shown in Figure 9, eight hall sensors are distributed evenly on a circle of radius $r$ and center $\mathrm{O}$ at locations $\mathrm{a}, \mathrm{b}, \mathrm{c}, \mathrm{d}, \mathrm{e}, \mathrm{f}, \mathrm{g}$, and $\mathrm{h}$. When the primary conductor is shifted from $\mathrm{O}$ to $\mathrm{O}_{1}$, the off-center distance is $l$.

When the primary conductor is off-center, the magnetic field measured by the sensors at each point are $B_{a}, B_{b}, B_{c}, B_{d}, B_{e}, B_{f}, B_{g}$, and $B_{h}$, respectively, and the measured current can be calculated as:

$$
I_{1}=\frac{\left(B_{a}+B_{b}+B_{c}+B_{d}+B_{e}+B_{f}+B_{g}+B_{h}\right)}{8} \times 2 \pi r
$$




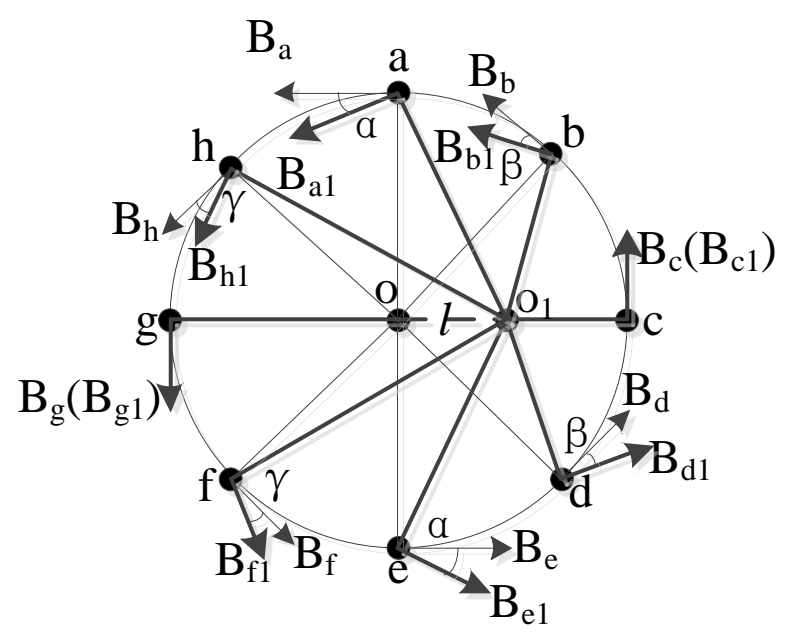

Figure 9. Schematic of an off-center primary conductor.

The measurement error is:

$$
I_{e 1}=\frac{I-I_{1}}{I} \times 100 \%
$$

From (10) and (11), the measurement error without any improvement approach can be given as:

$$
I_{e 1}=1-\left(\frac{2 r^{2}-\sqrt{2} r l}{2 \pi r\left(r^{2}+l^{2}-\sqrt{2} r l\right)}+\frac{2 r^{2}+\sqrt{2} r l}{2 \pi r\left(r^{2}+l^{2}+\sqrt{2} r l\right)}+\frac{r}{\pi\left(r^{2}+l^{2}\right)}+\frac{1}{2 \pi(r-l)}+\frac{1}{2 \pi(r+l)}\right) \times \frac{\pi r}{4}
$$

Suppose the radius of the circle is $r=0.1 \mathrm{~m}$ and the maximum value of the off-center distance $l$ is $5 \mathrm{~mm}$. Then, the measurement error as a function of the off-center distance can be plotted as shown in Figure 10. It can be seen that the error increases as the off-center distance increases. The error is larger than $0.6 \%$ when the off-center distance is $5 \mathrm{~mm}$.

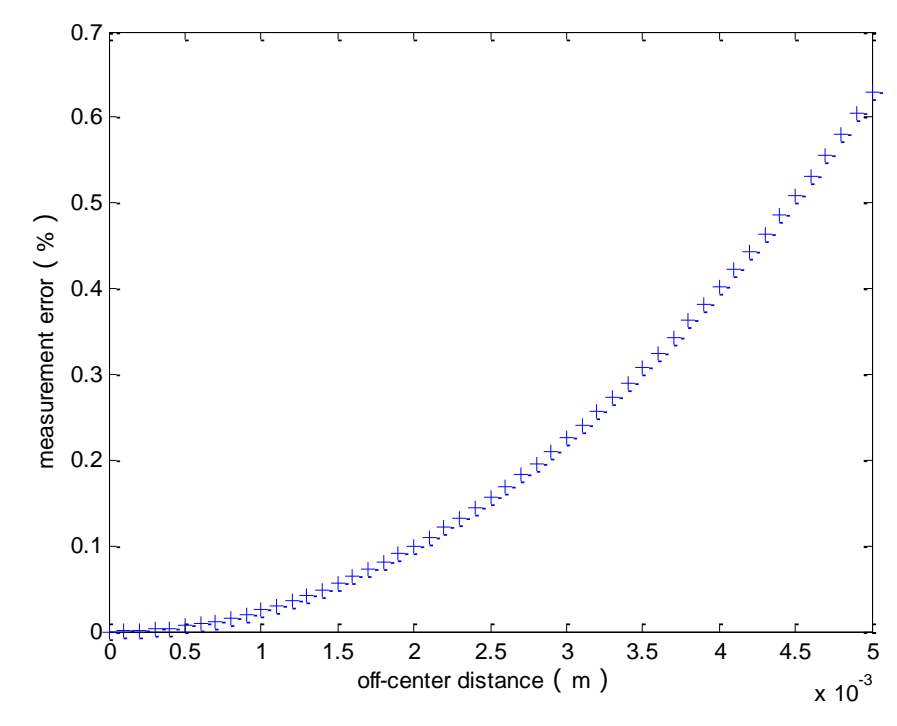

Figure 10. The measurement error verses the off-center distance.

It is obvious from Figure 10 and Equation (11) that the measured current $I_{1}$ is less than the real current $I$ due to the error introduced by the off-center distance. From Figure 10, one can conclude that if the magnetic field strength measured by magnetic field sensors is stronger, the measured current $I_{1}$ will be larger and closer to the real current $I$. Hence, a compensation method for the magnetic field 
strength is proposed in this study to improve the measurement accuracy when the primary conductor is off-center. The detailed analysis is as follows.

As shown in Figure 9, when the conductor is off-center, taking point a as an example, the magnetic field strength measured at point a is $B_{a}$. To make $B_{a}$ larger and measurement error smaller, we compensated for the magnetic field strength at point a, turning $B_{a}$ into $B_{a 1}$.

As shown in Figure 11, the relationship between $B_{a}$ and $B_{a 1}$ is:

$$
B_{a 1}=\frac{B_{a}}{\cos \alpha}
$$

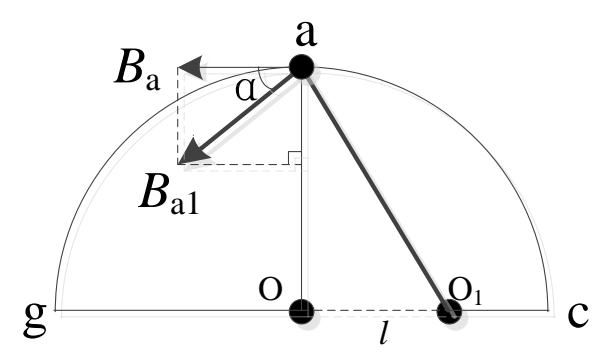

Figure 11. Schematic diagram of $B_{a}$ and $B_{a 1}$.

For the eight points sensors locations in Figure 9, the compensated magnetic fields can be written as:

$$
\left\{\begin{array}{l}
B_{a 1}=\frac{B_{a}}{\cos \alpha} \\
B_{b 1}=\frac{B_{b}}{\cos \beta} \\
B_{c 1}=B_{c} \\
B_{d 1}=\frac{B_{d}}{\cos \beta} \\
B_{e 1}=\frac{B_{e}}{\cos \alpha} \\
B_{f 1}=\frac{B_{f}}{\cos \gamma} \\
B_{g 1}=B_{g} \\
B_{h 1}=\frac{B_{h}}{\cos \gamma}
\end{array}\right.
$$

In (13) and (14), the distance $l$ can be obtained by the magnetic field strength at points $\mathrm{c}$ and $\mathrm{g}$, after which the angles of $\alpha, \beta$, and $\gamma$ can be calculated by using the radius $r$.

By using this compensation method, the improved equation for the measured current can be given as:

$$
I_{2}=\frac{\left(B_{a 1}+B_{b 1}+B_{c 1}+B_{d 1}+B_{e 1}+B_{f 1}+B_{g 1}+B_{h 1}\right)}{8} \times 2 \pi r
$$

The measurement error is:

$$
I_{e 2}=\frac{I-I_{2}}{I} \times 100 \%
$$

From (15) and (16), the measurement error using the proposed improvement approach can be given as:

$$
I_{e 2}=1-\frac{\pi r}{4} \times\left(\frac{1}{\pi \sqrt{l^{2}+r^{2}}}+\frac{1}{\pi \sqrt{\left(\frac{\sqrt{2}}{2} r-l\right)^{2}+\left(\frac{\sqrt{2}}{2} r\right)^{2}}}+\frac{1}{\pi \sqrt{\left(\frac{\sqrt{2}}{2} r+l\right)^{2}+\left(\frac{\sqrt{2}}{2} r\right)^{2}}}+\frac{1}{2 \pi(r-l)}+\frac{1}{2 \pi(r+l)}\right)
$$

Figure 12 shows a comparison of the measurement error as a function of the off-center distance with and without the proposed compensation approach. By adopting the proposed compensation approach, the measurement error can be effectively reduced to a level less than $0.1 \%$, even with a maximum off-center distance. 


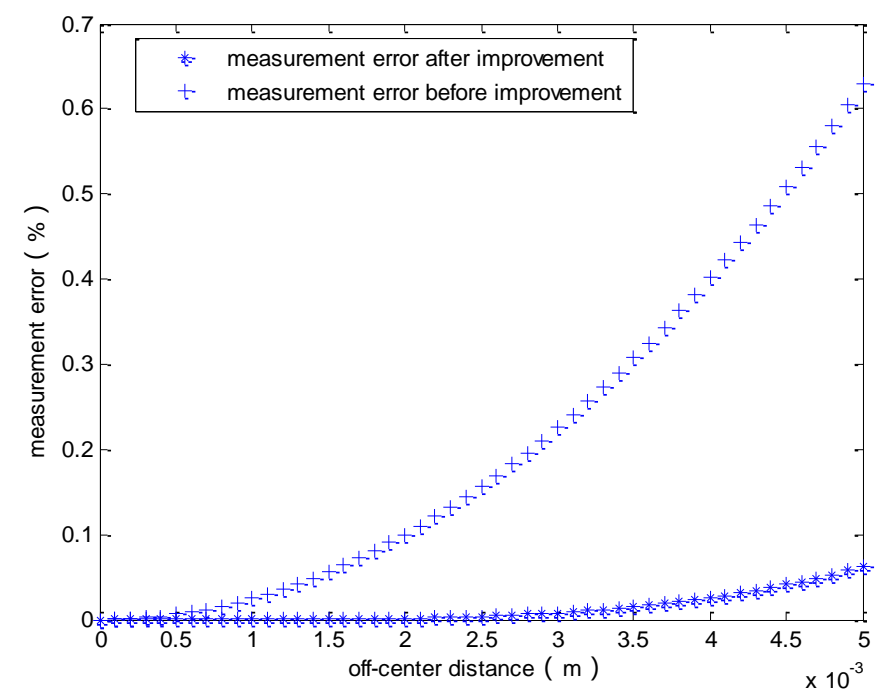

Figure 12. Comparison of the measurement errors without and with the compensation approach.

\section{Performance Test}

To assess the robustness of the proposed approach, a circular magnetic field sensor array with a radius of $0.1 \mathrm{~m}$ and eight hall sensors distributed evenly on a circle was developed and subjected to the below analysis. The type of the hall sensor employed was EQ-730L, produced by AKM, Tokyo, Japan, which has wide range of measurement and high sensitivity.

\subsection{Influence of Magnetic Field due to a Parallel Conductor}

The developed sensor array was utilized to measure a current of $600 \mathrm{~A}$. The overall measured value and the measurement error for various distances between the two conductors are shown in Figure 13. It can be seen that when the distance was larger than $0.237 \mathrm{~m}$, i.e., when $d / r$ was larger than 2.37, the measurement error was less than $0.1 \%$. In the actual situation, the distance between the two conductors is much larger than $0.237 \mathrm{~m}$, so the accuracy can meet the requirements of the 0.1 accuracy class.

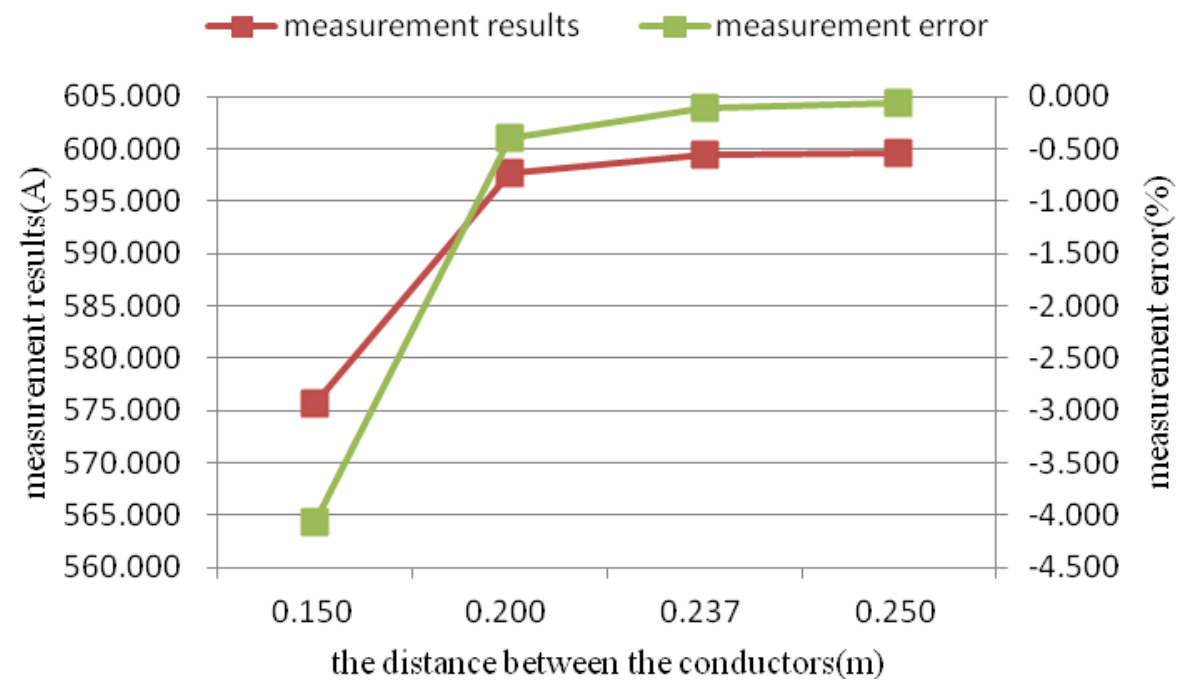

Figure 13. Measured results verses the distance between the conductors. 


\subsection{Off-Center Conductor Analysis}

Figure 14 shows the possible alignment of the primary conductor that could be perfectly aligned at the center of the sensor array or off-center. Measurements were conducted when the primary conductor was off-center, and the results are listed in Table 1. Without the proposed compensation approach, it can be seen that the measurement error was larger than $0.6 \%$; however, it was reduced to $0.1 \%$ by the proposed approach.

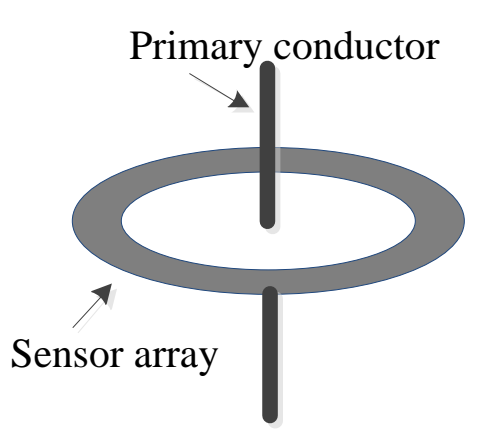

(a)

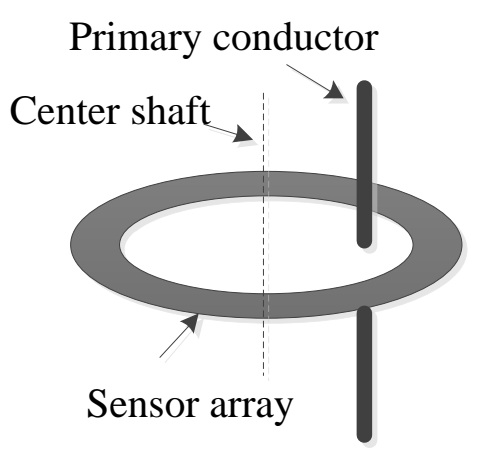

(b)

Figure 14. A schematic diagram for the primary conductor alignment with respect to the sensor array: (a) primary conductor is on the array's center; (b) primary conductor is off-center.

Table 1. Measurement results for various off-center distances before and after improvement.

\begin{tabular}{cccccc}
\hline $\begin{array}{c}\text { Current of } \\
\text { Primary } \\
\text { Conductor (A) }\end{array}$ & $\begin{array}{c}\text { Off-Center } \\
\text { Distance (mm) }\end{array}$ & $\begin{array}{c}\text { Measurement } \\
\text { Results before } \\
\text { Improvement (A) }\end{array}$ & $\begin{array}{c}\text { Measurement } \\
\text { Results after } \\
\text { Improvement (A) }\end{array}$ & $\begin{array}{c}\text { Measurement } \\
\text { Error before } \\
\text { Improvement (\%) }\end{array}$ & $\begin{array}{c}\text { Measurement } \\
\text { Error after } \\
\text { Improvement (\%) }\end{array}$ \\
\hline 600 & 1 & 599.85 & 599.99 & 0.025 & 0.001 \\
600 & 2 & 599.34 & 599.93 & 0.110 & 0.012 \\
600 & 3 & 598.74 & 599.89 & 0.210 & 0.019 \\
600 & 4 & 597.42 & 599.78 & 0.430 & 0.036 \\
600 & 5 & 596.16 & 599.63 & 0.640 & 0.062 \\
\hline
\end{tabular}

\subsection{Basic Accuracy Test}

A basic accuracy test of the proposed current measurement method was carried out to measure the wide range of current levels. Figure 15 shows that the measurement error was less than $0.1 \%$ when the range of current was $20 \%$ to $120 \%$ of the investigated rated current $(600 \mathrm{~A})$.

Uncertainty in the measurements can be calculated as:

$$
\left\{\begin{array}{l}
s=\sqrt{\frac{\sum_{k=1}^{N}\left(I_{\varepsilon k}-\overline{I_{\varepsilon}}\right)^{2}}{N-1}} \\
u=\frac{s}{\sqrt{N}}
\end{array}\right.
$$

where $s$ is the experimental standard deviations, $N$ is the number of tests, $I_{\varepsilon k}$ is the measurement error of the $k$ th test, $\overline{I_{\varepsilon}}$ is the average of the all measurement errors, and $u$ is the measurement uncertainty.

Measurements of the $600 \mathrm{~A}$ current were repeated 10 times, and the uncertainty in the measurements was found to be only $0.033 \%$. 


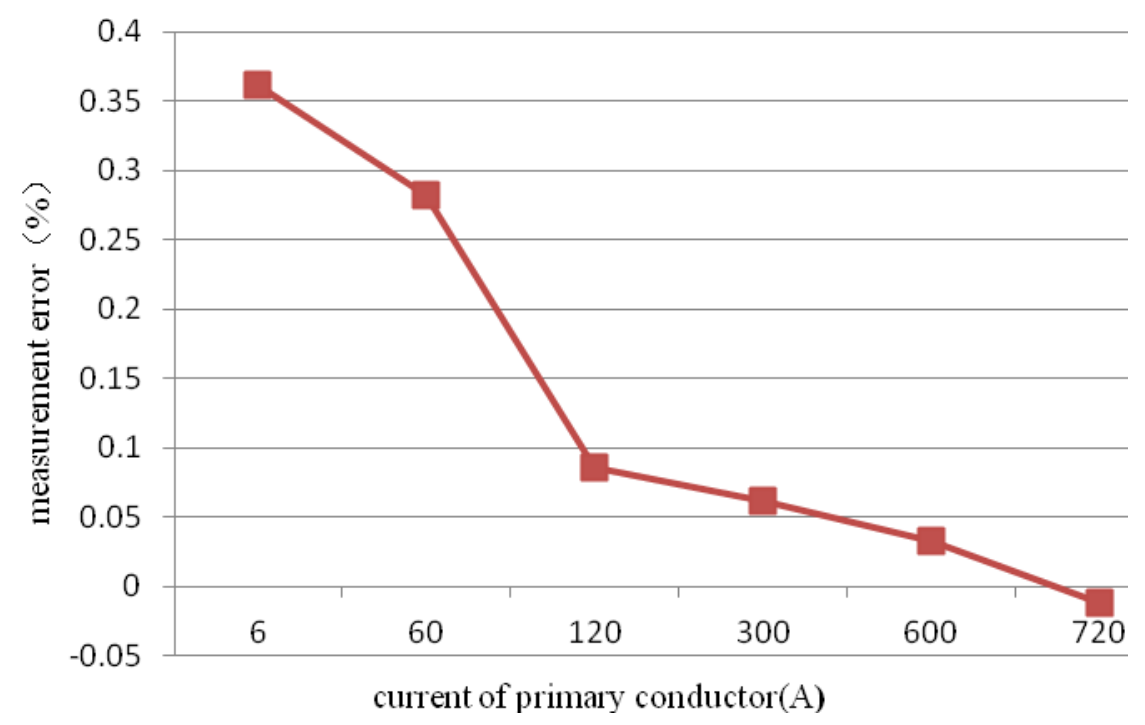

Figure 15. Basic accuracy test.

\section{Conclusions}

In order to overcome the deficiency of conventional transformers and improve the accuracy of DC measurements, this paper proposes a current measurement method based on a circular magnetic field sensing array. By employing eight hall sensors distributed evenly on a circle and using the average value of these hall sensors as the final measured value, the interference generated by the external magnetic field can be effectively reduced and the measurement accuracy can be improved. Also, a kind of hall sensor compensation method is adopted to reduce the measurement error to less than $0.1 \%$.

Author Contributions: Conceptualization: Zhenhua Li, Siqiu Zhang, Zhengtian Wu, and Yuan Tao; Data curation: Zhenhua Li, Siqiu Zhang, Zhengtian Wu, and Yuan Tao; Formal analysis: Zhenhua Li, Siqiu Zhang, Zhengtian Wu, and Yuan Tao; Methodology: Ahmed Abu-Siada; Writing-original draft: Zhenhua Li, Siqiu Zhang, Zhengtian Wu, and Yuan Tao; Writing-review and editing: Ahmed Abu-Siada.

Funding: This research was funded by the China Scholarship Council and National Natural Science Foundation of China: 51507091.

Conflicts of Interest: The authors declare no conflict interest.

\section{References}

1. Matsumoto, H.; Shibako, Y.; Shiihara, Y.; Nagata, R.; Neba, Y. Three-phase lines to Single-phase Coil Planar Contactless Power Transformer. IEEE Trans. Ind. Electron. 2018, 65, 2904-2914. [CrossRef]

2. Dave, M.; Bhagdev, M. Design and Analysis of Adaptable Power Electronic Transformer. In Proceedings of the 2017 2nd IEEE International Conference on Electrical Computer and Communication Technology (ICECCT), Coimbatore, India, 22-24 February 2017; pp. 1-6.

3. Sabahi, M.; Hosseini, S.H.; Sharifian, M.B.B.; Goharrizi, A.Y.; Gharehpetian, G.B. A three-phase dimmable lighting system using a bidirectional power electronic transformer. IEEE Trans. Power Electron. 2009, 24, 830-837. [CrossRef]

4. Sabahi, M.; Goharrizi, A.Y.; Hosseini, S.H.; Sharifian, M.B.B.; Gharehpetian, G.B. Flexible Power Electronic Transformer. IEEE Trans. Power Electron. 2010, 25, 2159-2169. [CrossRef]

5. Zhao, J.Y.; Yao, F.; Mi, Y.Z.; Qin, N. Research on Optimization Technology of Electromagnetic Transformer. Energy Power Technol. 2013, 805, 818-821. [CrossRef]

6. Warrier, P.V.; Preetha, P.K. Electromagnetic analysis of transformer using Solid works. In Proceedings of the 2015 International Conference on Technological Advancements in Power and Energy (TAP Energy), Kollam, India, 24-26 June 2015; pp. 427-431. 
7. Li, Z.H.; Yan, S.H.; Hu, W.Z. High Accuracy On-Line Calibration System for Current Transformers Based on Clamp-Shape Rogowski Coil and Improved Digital Integrator. Mapan-J. Metrol. Soc. India 2016, 31, 119-127. [CrossRef]

8. Li, Z.H.; Hu, W.Z. A high-precision digital integrator based on the Romberg algorithm. Rev. Sci. Instrum. 2017, 88, 138-142. [CrossRef] [PubMed]

9. Dubickas, V.; Edin, H. High-Frequency Model of the Rogowski Coil with a Small Number of Turns. IEEE Trans. Instrum. Meas. 2007, 56, 2284-2288. [CrossRef]

10. Lehtonen, T.A.; Hällström, J. Optimizing Temperature Coefficient and Frequency Response of Rogowski Coils. IEEE Sens. J. 2017, 17, 6646-6651. [CrossRef]

11. Oosthuysen, N.J.; Walker, J.J. Optimizing the Properties of the Optical Current Transformer with Special Reference to faster transient response. In Proceedings of the International Conference on High Voltage Engineering and Application, Poznan, Poland, 8-11 September 2014; pp. 1-4.

12. Ashraf, H.M.; Abbas, G.; Ali, U.; Rehman, S.U. Modeling and Simulation of Optical Current Transformer using operational amplifiers. In Proceedings of the 5th International Conference on Electrical Engineering(ICEE), Boumerdes, Algeria, 29-31 October 2017; pp. 1-6.

13. Araujo, Z.; Davila, M.; Mora, E.; Maldonado, L.; Ferraz, G. Analysis of the Behavior of an Optical Current Transformer using an Equivalent circuit. In Proceedings of the 6th Andean Region International Conference, Cuenca, Ecuador, 7-9 November 2012; pp. 81-84.

14. Sasaki, K.; Takahashi, M.; Hirata, Y. Temperature-Insensitive Sagnac-Type Optical Current Transformer. J. Lightwave Technol. 2015, 33, 2463-2467. [CrossRef]

15. Santos, J.C.; Sillos, A.C.; Nascimento, C.G.S. On-Field Instrument Transformers Calibration Using Optical Current and Voltage Transformers. In Proceedings of the IEEE International Workshop on Applied Measurements for Power Systems (AMPS), Aachen, Germany, 24-26 September 2014; pp. 1-5.

16. Santos, J.C.; Almeida, J.C.J.; Silva, L.P.C. White Light Interferometry High Voltage Optical Fiber Sensor Systems with Compensation for Optical Power Fluctuations. In Proceedings of the Third European Workshop on Optical Fibre Sensors, Napoli, Italy, 4-6 July 2007; pp. 1-5.

17. Samimi, M.H.; Bahrami, S.; Akmal, A.A.S.; Mohseni, H. Effect of Nonideal Linear Polarizers, Stray Magnetic Field, and Vibration on the Accuracy of Open-Core Optical Current Transducers. IEEE Sens. J. 2014, 14, 3508-3515. [CrossRef]

18. Ouyang, Y.; He, J.L.; Hu, J.; Wang, S.X. A Current Sensor Based on the Giant Magnetoresistance Effect: Design and Potential Smart Grid Applications. Sensors 2012, 12, 15520-15541. [CrossRef] [PubMed]

19. Ziegler, S.; Woodward, R.C.; Iu, H.H.C.; Borle, L.J. Current Sensing Techniques: A Review. IEEE Sens. J. 2009, 9, 354-376. [CrossRef]

20. Andrea, M.H.; Marc, P.; Maher, K. A Fully Integrated Hall Sensor Microsystem for Contactless Current Measurement. IEEE Sens. J. 2013, 13, 2271-2278. [CrossRef]

21. Nama, T.; Gogoi, A.K.; Tripathy, P. Application of a Smart Hall Effect Sensor System for 3-Phase BLDC Drives. In Proceedings of the IEEE 5th International Symposium on Robotics and Intelligent Sensors(IRIS), Ottawa, ON, Canada, 5-7 October 2017; pp. 1-28.

22. Fernandez, D.; Hyun, D.; Park, Y.; Reigosa, D.D.; Lee, S.B.; Lee, D.M.; Briz, F. Permanent Magnet Temperature Estimation in PM Synchronous Motors Using Low-Cost Hall Effect Sensors. IEEE Trans. Ind. Appl. 2017, 53, 4515-4525. [CrossRef]

23. Song, K.J.; Lim, J.C.; Ko, R.K.; Park, C.; Schwartz, J. A Noncontact $T_{c}$ Evaluation Technique Using a Hall Probe Array. IEEE Trans. Appl. Superconduct. 2017, 27, 1-4. [CrossRef]

24. Jiang, J.F.; Makinwa, K.A.A. Multipath Wide-Bandwidth CMOS Magnetic Sensors. IEEE J. Solid State Circuits 2017, 52, 198-209. [CrossRef]

25. Sen, M.; Balabozov, I.; Yatchev, I.; Ivanov, R. Modelling of Current Sensor Based on Hall Effect. In Proceedings of the 2017 15th International Conference on Electrical Machines, Drives and Power Systems (ELMA), Yekaterinburg, Russia, 1-3 June 2017; pp. 457-460.

26. Li, C.S.; Cui, H.; Zhang, X. Optical Magnetic Field Sensor Based on Electrogyratory and Electrooptic Compensation in Single Quartz Crystal. IEEE Sens. J. 2018, 18, 1427-1434. [CrossRef]

27. Salzer, S.; Röbisch, V.; Klug, M.; Durdaut, P.; McCord, J.; Meyners, D.; Reermann, J.; Höft, M.; Knöchel, R. Noise Limits in Thin-Film Magnetoelectric Sensors With Magnetic Frequency Conversion. IEEE Sens. J. 2018, 18, 596-604. [CrossRef] 
28. Bazzocchi, R.; Rienzo, L.D. Interference Rejection Algorithm for Current Measurement using Magnetic Sensors Array. Sens. Actuators A. Phys. 2000, 85, 38-41. [CrossRef]

29. Rienzo, L.D.; Bazzocchi, R.; Manara, A. Circular Arrays of Magnetic Sensors for Current Measurement. IEEE Trans. Instrum. Meas. 2001, 50, 1093-1096. [CrossRef]

(C) 2018 by the authors. Licensee MDPI, Basel, Switzerland. This article is an open access article distributed under the terms and conditions of the Creative Commons Attribution (CC BY) license (http:/ / creativecommons.org/licenses/by/4.0/). 